\title{
Partitioning Balls into Topologically Equivalent Pieces
}

\author{
Christian Richter \\ Christian Richter wurde 1969 in Jena geboren. Nach dem Abitur studierte er Ma- \\ thematik an der Friedrich-Schiller-Universität in Jena. Gegenwärtig ist er wissen- \\ schaftlicher Assistent am dortigen mathematischen Institut und beschäftigt sich in \\ Vorbereitung auf eine Habilitation mit Zerlegungs- und Überdeckungsproblemen me- \\ trischer Räume und mit approximationstheoretischen Anwendungen. In seiner Frei- \\ zeit unternimmt er gerne Wanderungen und findet Entspannung beim Musikhören.
}

\section{Introduction}

In 1949 B. L. van der Waerden asked for a proof for the non-existence of a disjoint decomposition $B=A_{1} \cup A_{2}$ of a closed ball (circle) in the Euclidean plane $\mathbb{E}^{2}$ into two congruent pieces $A_{1}$ and $A_{2}$ (cf. [Wae]). This problem found, beside its solution in the same issue of "Elemente der Mathematik", many generalizations (cf. [Wa1], [Wa2], [He], [Ede1], [Ede2], [E/J/T], [Ri]). We use the most general notation from [Wa1]: Let $\mathscr{G}$ be a group acting on a set $X$. A subset $Y \subseteq X$ is called $n$-divisible w.r.t. $\mathscr{G}$ if $Y$ may be partitioned into $n$ pieces which are pairwise congruent via $\mathscr{G}$.

Im Jahre 1949 veröffentlichten die Elemente der Mathematik eine Aufgabe von van der Waerden:

Ist es möglich, eine Kreisscheibe in zwei zueinander fremde kongruente Punktmengen zu zerlegen? (Ob man den Randkreis zur Kreisscheibe rechnet oder nicht, ist gleichgültig. Das einfachste ist, den Randkreis dazu zu rechnen.)

In den vergangenen 50 Jahren wurden verschiedene Verallgemeinerungen dieses Problems behandelt. Sie betreffen insbesondere den Fall höherer Dimensionen und den Fall, wo statt kongruenter Teile topologisch äquivalente Teile betrachtet werden. Christian Richter beweist im vorliegenden Beitrag das folgende allgemeine Resultat:

Eine abgeschlossene Kugel B im d-dimensionalen euklidischen Raum lässt genau dann eine Zerlegung in $n$ topologisch äquivalente Teile $z u$, wenn das Paar $(d, n)$ vom Paar $(1,2)$ verschieden ist. ust 
In the Euclidean space $\mathbb{E}^{d}$ it is the natural problem to discuss the $n$-divisibility of sets w.r.t. the group $\Re^{d}$ of all isometries of $\mathbb{E}^{d}$. In the paper $[\mathrm{He}]$ it is shown that every closed, bounded and convex set in $\mathbb{E}^{d}$ is not 2-divisible w.r.t. $\mathscr{B}^{d}$. Moreover it is proved that closed intervals are not $n$-divisible w.r.t. $\mathscr{B}^{1}$ for all $n \in\{2,3,4, \ldots\}$. E. Hertel asks for the largest group of transformations of $\mathbb{E}^{d}$ for which these results can be generalized.

Of course, half-open intervals $[a, b) \subseteq \mathbb{E}^{1}$ are $n$-divisible w.r.t. translations. Similarly, the small manipulation of removing its center makes a circle in the Euclidean plane $n$-divisible w.r.t. rotations. This shows that "small" topological changes can change the property of $n$-divisibility of sets. On that account it becomes interesting to consider the $n$-divisibility of bounded convex sets w.r.t. the group $\mathscr{T}^{d}$ of all homeomorphisms of $\mathbb{E}^{d}$ onto itself. It is obviously sufficient to restrict the considerations to balls, which represent the topological type of bounded convex sets. We call a set $B \subseteq \mathbb{E}^{d}$ a (general) ball of radius $r>0$ centered in $x_{0} \in \mathbb{E}^{d}$ if

$$
\left\{x \in \mathbb{E}^{d}:\left\|x-x_{0}\right\|<r\right\} \subseteq B \subseteq\left\{x \in \mathbb{E}^{d}:\left\|x-x_{0}\right\| \leq r\right\} .
$$

\section{Partitioning closed balls}

Before we discuss the $n$-divisibility of closed balls in $\mathbb{E}^{d}$, we ask for partitions into $n$ pairwise homeomorphic topological subspaces. This property of the partition sets is more general than the pairwise congruence via $\mathscr{T}^{d}$, since a homeomorphic bijection between two subsets of $\mathbb{E}^{d}$ can not be extended to a transformation from $\mathscr{T}^{d}$ in general.

Theorem 1 Let $B$ be a closed ball in $\mathbb{E}^{d}(d \geq 1)$ and let $n \geq 2$. Then there exists a disjoint decomposition of $B$ into $n$ pairwise homeomorphic topological subspaces of $\mathbb{E}^{d}$.

Proof. The cases $(d, n) \neq(1,2)$ will be discussed in the proof of the following Theorem 2.

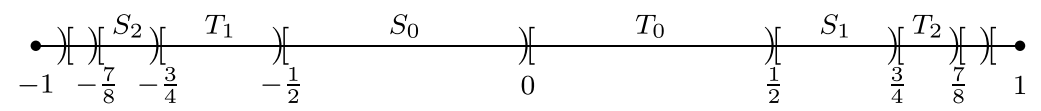

Fig. 1

If $d=1$ and $n=2$ we assume that $B=[-1,1]$ without loss of generality (cf. Fig. 1). We consider the decomposition $B=S \cup T$ where

$$
S=\{-1\} \cup \bigcup_{k=0}^{\infty} S_{k} \quad \text { with } \quad S_{k}= \begin{cases}{\left[-1+2^{-(k+1)},-1+2^{-k}\right)} & \text { if } k \text { is even, } \\ {\left[1-2^{-k}, 1-2^{-(k+1)}\right)} & \text { if } k \text { is odd }\end{cases}
$$

and

$$
T=\{1\} \cup \bigcup_{k=0}^{\infty} T_{k} \quad \text { with } \quad T_{k}= \begin{cases}{\left[1-2^{-k}, 1-2^{-(k+1)}\right)} & \text { if } k \text { is even, } \\ {\left[-1+2^{-(k+1)},-1+2^{-k}\right)} & \text { if } k \text { is odd. }\end{cases}
$$


A homeomorphism $\varphi$ from $S$ onto $T$ can be obtained as follows: We define the restriction $\left.\varphi\right|_{S_{k}}$ of $\varphi$ to the interval $S_{k}, k \in\{0,1,2, \ldots\}$, to be the translation of $S_{k}$ onto $T_{k}$ and, of course, $\varphi(-1)=1$. The continuity of $\varphi$ in a point $x_{0} \in S \backslash\{-1\}$, say $x_{0} \in S_{k_{0}}$, is implied by the existence of a neighbourhood $U\left(x_{0}\right)$ in the topological space $S$ such that $U\left(x_{0}\right) \subseteq S_{k_{0}}$. Hence $\left.\varphi\right|_{U\left(x_{0}\right)}$ is a restriction of a translation and, consequently, $\varphi$ is continuous in $x_{0}$. If $x_{0}=-1$ we observe, that all points $x \in S$ with $\left\|x-x_{0}\right\| \leq 2^{-2 i}$, i.e. $x \in\{-1\} \cup \bigcup_{l=i}^{\infty} S_{2 l}$, are mapped into $\{1\} \cup \bigcup_{l=i}^{\infty} T_{2 l}$, i.e. $\left\|\varphi(x)-\varphi\left(x_{0}\right)\right\|=\|\varphi(x)-1\| \leq 2^{-2 i}$. The continuity of $\varphi^{-1}$ can analogously be checked. This completes the proof.

The investigation of the $n$-divisibility w.r.t. $\mathscr{T}^{d}$ requires several steps. The first proposition shows that this $n$-divisibility is strictly harder than the partition property considered in Theorem 1. (Proposition 1 is proved in a more general context in [Ri]. In the present paper we give an elementary proof.)

Proposition 1 Closed balls of $\mathbb{E}^{1}$ are not 2-divisible w.r.t. $\mathscr{T}^{1}$.

Proof. Without loss of generality we consider the ball $B=[0,1]$. We assume the contrary, i.e. there exist two disjoint sets $S$ and $T$ and a homeomorphism $\tau \in \mathscr{T}^{1}$ such that

$$
[0,1]=S \cup T, \quad \tau(S)=T \quad \text { and } \quad 0 \in S .
$$

Fact 1: $\tau$ has no fixed point $x_{0}=\tau\left(x_{0}\right)$ in $[0,1]$.

Such a point would belong to both the sets $S$ and $T$, since $\tau(S)=T$, in contradiction to the disjointness of $S$ and $T$.

Fact 2: $\tau$ is either strictly increasing or strictly decreasing.

This is simply implied by the injectivity of $\tau$ and Cauchy's intermediate value theorem.

Case 1: $\tau$ is strictly increasing.

We prove the following statement by induction w.r.t. $i$ :

Fact 3: Let $i \in\{0,1,2, \ldots\}$. Then

$$
\begin{aligned}
& \tau^{2 i}(0)<\tau^{2 i+1}(0)<\tau^{2 i+2}(0), \\
& {\left[\tau^{2 i+2}(0), 1\right]=\left(S \cap\left[\tau^{2 i+2}(0), 1\right]\right) \cup\left(T \cap\left[\tau^{2 i+2}(0), 1\right]\right),} \\
& \tau\left(S \cap\left[\tau^{2 i+2}(0), 1\right]\right)=T \cap\left[\tau^{2 i+2}(0), 1\right] \quad \text { and } \\
& \tau^{2 i+2}(0) \in S \cap\left[\tau^{2 i+2}(0), 1\right] .
\end{aligned}
$$

We start with $i=0$. The first inequality from $\left(\alpha_{0}\right)$ is easily seen, since

$$
\tau^{1}(0) \in \tau(S)=T=[0,1] \backslash S \subseteq(0,1]
$$

and thus $\tau^{0}(0)=0<\tau^{1}(0)$. Application of $\tau$ to this inequality yields $\tau^{1}(0)<\tau^{2}(0)$, which completes the verification of $\left(\alpha_{0}\right)$. Equation $\left(\beta_{0}\right)$ is trivial in accordance with (1). Moreover we have

$$
\min (T)=\min (\tau(S))=\tau(\min (S))=\tau(0)
$$


hence

$$
[0, \tau(0)) \subseteq S
$$

and, by application of $\tau$,

$$
\left[\tau(0), \tau^{2}(0)\right) \subseteq T
$$

These inclusions imply that

$$
S \cap\left[0, \tau^{2}(0)\right)=[0, \tau(0)) \quad \text { and } \quad T \cap\left[0, \tau^{2}(0)\right)=\left[\tau(0), \tau^{2}(0)\right) .
$$

We obtain $\left(\gamma_{0}\right)$ by

$$
\begin{aligned}
\tau\left(S \cap\left[\tau^{2}(0), 1\right]\right) & =\tau\left(S \backslash\left[0, \tau^{2}(0)\right)\right) \\
& =\tau(S \backslash[0, \tau(0))) \\
& =T \backslash\left[\tau(0), \tau^{2}(0)\right) \\
& =T \backslash\left[0, \tau^{2}(0)\right) \\
& =T \cap\left[\tau^{2}(0), 1\right] .
\end{aligned}
$$

Finally, we have $\tau^{2}(0) \in[0,1]$, since $\tau^{2}(0)$ belongs to the closure of $T$ in accordance with the second part of (2). If $\tau^{2}(0)$ would belong to $T$ we would obtain

$$
\tau(0)=\tau^{-1}\left(\tau^{2}(0)\right) \in \tau^{-1}(T)=S
$$

in contradiction to (2). This shows the inclusion $\tau^{2}(0) \in S$ and completes the verification of $\left(\delta_{0}\right)$.

The step from $i-1$ to $i, i \geq 1$, can similarly be done. We presuppose $\left(\beta_{i-1}\right),\left(\gamma_{i-1}\right)$, and $\left(\delta_{i-1}\right)$ instead of (1), i.e. we replace $[0,1]$ by $\left[\tau^{2 i}(0), 1\right], S$ by $S \cap\left[\tau^{2 i}(0), 1\right]$, $T$ by $T \cap\left[\tau^{2 i}(0), 1\right]$, and 0 by $\tau^{2 i}(0)$. Then we infer $\left(\alpha_{i}\right),\left(\beta_{i}\right),\left(\gamma_{i}\right)$, and $\left(\delta_{i}\right)$ by the same arguments as above. This completes the proof of Fact 3.

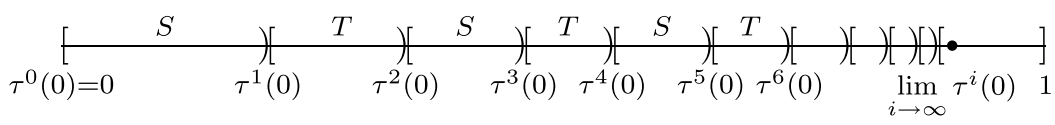

Fig. 2

According to Fact 3 we have an increasing sequence $\left(\tau^{i}(0)\right)_{i=0}^{\infty}$ in $[0,1]$ (cf. Fig. 2). The limit $x_{0} \in[0,1]$ of this sequence is a fixed point of $\tau$, since

$$
\tau\left(x_{0}\right)=\tau\left(\lim _{i \rightarrow \infty} \tau^{i}(0)\right)=\lim _{i \rightarrow \infty} \tau^{i+1}(0)=x_{0} .
$$

Hence we obtained a contradiction to Fact 1 . This closes the considerations of the first case.

Case 2: $\tau$ is strictly decreasing.

We have $\tau(0) \in \tau(S) \subseteq[0,1]$. According to Fact 1 we obtain $0<\tau(0) \leq 1$. Moreover we have $\tau(1)<\tau(0) \leq 1$. Consequently, $\tau(0)-\mathrm{id}(0)>0$ and $\tau(1)-\mathrm{id}(1)<0$. Hence there exists an $x_{0} \in(0,1)$ with $\tau\left(x_{0}\right)-x_{0}=0$ in contradiction to Fact 1 . This completes the proof of Proposition 1 . 
Proposition 2 Every closed ball in $\mathbb{E}^{1}$ is n-divisible w.r.t. $\mathscr{T}^{1}$ for all integers $n \in$ $\{3,4,5, \ldots\}$.

Proof. We consider the ball $B=[0, n]$, which can be decomposed into the $n$ intervals $[0,1),[1,2), \ldots,[n-2, n-1),[n-1, n]$ (cf. Fig. 3 ). The half-open intervals $[j-1, j$ ), $1 \leq j \leq n-1$, possess a partition

$$
\begin{aligned}
{[j-1, j) } & =S_{j} \cup T_{j} \quad \text { with } \\
S_{j} & =\left\{j-\frac{1}{2}\right\} \cup \bigcup_{k=1}^{\infty}\left(\left\{j-\frac{1}{2}\right\}+(-1)^{k} \cdot\left[2^{-k-1}, 2^{-k}\right]\right), \\
T_{j} & =\bigcup_{k=1}^{\infty}\left(\left\{j-\frac{1}{2}\right\}+(-1)^{k+1} \cdot\left(2^{-k-1}, 2^{-k}\right)\right) .
\end{aligned}
$$

The remaining interval $[n-1, n]$ can be decomposed into

$$
\begin{aligned}
{[n-1, n] } & =S_{n} \cup T_{n} \quad \text { with } \\
S_{n} & =\left\{n-\frac{1}{2}\right\} \cup \bigcup_{k=1}^{\infty}\left(\left\{n-\frac{1}{2}\right\}+\left[-2^{-2 k+1},-2^{-2 k}\right] \cup\left[2^{-2 k}, 2^{-2 k+1}\right]\right), \\
T_{n} & =\bigcup_{k=1}^{\infty}\left(\left\{n-\frac{1}{2}\right\}+\left(-2^{-2 k},-2^{-2 k-1}\right) \cup\left(2^{-2 k-1}, 2^{-2 k}\right)\right) .
\end{aligned}
$$

We consider the following partition:

$$
\begin{aligned}
B & =A_{1} \cup A_{2} \cup \ldots \cup A_{n} \quad \text { with } \\
A_{j} & = \begin{cases}S_{j} \cup T_{j+1} & \text { if } 1 \leq j \leq n-1, \\
S_{n} \cup T_{1} & \text { if } j=n\end{cases}
\end{aligned}
$$

All the sets $A_{1}, A_{2}, \ldots, A_{n-1}$ and the reflection $-A_{n}$ of $A_{n}$ have the following topological structure:

$$
A=\left(\bigcup_{i=1}^{\infty} \hat{C}_{i}\right) \cup\{c\} \cup\left(\bigcup_{i=1}^{\infty} \check{C}_{i}\right) \cup\left(\bigcup_{i=1}^{\infty} \hat{O}_{i}\right) \cup\left(\bigcup_{i=1}^{\infty} \check{O}_{i}\right)
$$

The subsets $\hat{C}_{i}$ form an increasing sequence of closed and bounded intervals of positive length which tends to a point $c \in A .\left(\check{C}_{i}\right)_{i=1}^{\infty}$ is a decreasing sequence of closed and bounded intervals of positive length tending to $c$ from above. Similarly, the sets $\hat{O}_{i}$ are open and bounded intervals of positive length which accumulate in $o \notin A$ from below. The sequence $\left(\breve{O}_{i}\right)_{i=1}^{\infty}$ consists of open and bounded intervals of positive length and tends to $o$ from above. All the intervals $\hat{C}_{i}, \check{C}_{i}, \hat{O}_{i}$, and $\check{O}_{i}$ are pairwise separated by intervals of positive length. Moreover the closed set consisting of $\{c\}$ and the closed intervals is separated by an interval from the open set formed by the open intervals, too. 


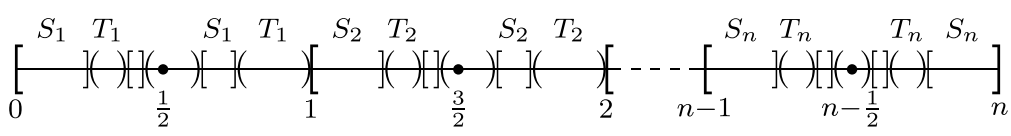

Fig. 3

For any two given sets $A$ and $A^{\prime}$ of that type there exists a strictly increasing homeomorphism $\tau \in \mathscr{T}^{1}$ such that $\tau(A)=A^{\prime}$. One can define $\tau$ piecewise linearly where $\hat{C}_{1}$ is mapped onto $\hat{C}_{1}^{\prime}, \hat{C}_{2}$ onto $\hat{C}_{2}^{\prime}, \ldots, c$ onto $c^{\prime}, \ldots, \breve{C}_{2}$ onto $\check{C}_{2}^{\prime}, \breve{C}_{1}$ onto $\breve{C}_{1}^{\prime}, \hat{O}_{1}$ onto $\hat{O}_{1}^{\prime}$, $\hat{O}_{2}$ onto $\hat{O}_{2}^{\prime}, \ldots, o$ onto $o^{\prime}, \ldots, \check{O}_{2}$ onto $\check{O}_{2}^{\prime}$, and $\check{O}_{1}$ onto $\check{O}_{1}^{\prime}$. This shows, that the sets $A_{i}, 1 \leq i \leq n$, are pairwise congruent via $\mathscr{T}^{1}$, and completes the proof of Proposition 2.

Let us remark that the above construction does not apply to the case $n=2$, since the two sets $S_{2}$ and $T_{1}$ would not be separated by an interval. This would change the topological structure of $A_{2}=S_{2} \cup T_{1}$.

Proposition 3 Every closed ball in $\mathbb{E}^{2}$ is 2-divisible w.r.t. $\mathscr{T}^{2}$.

Proof. We consider the rhomb $B=\left\{(x, y) \in \mathbb{E}^{2}:|x| \leq 1,|y| \leq \frac{1}{4}(1-|x|)\right\}$ instead of a ball (cf. Fig. 4). $B$ admits the decomposition $B=S \cup T$ where

$$
\begin{aligned}
& S=\{(-1,0)\} \cup \bigcup_{k=0}^{\infty} S_{k} \quad \text { and } \quad T=\{(1,0)\} \cup \bigcup_{k=0}^{\infty} T_{k} \quad \text { with } \\
& S_{k}=\left\{\begin{array}{ll}
\left\{(x, y) \in B: x \in\left[-1+2^{-(k+1)},-1+2^{-k}\right)\right\} & \text { if } k \text { is even, } \\
\left\{(x, y) \in B: x \in\left[1-2^{-k}, 1-2^{-(k+1)}\right)\right\} & \text { if } k \text { is odd, }
\end{array}\right. \text { and } \\
& T_{k}= \begin{cases}\left\{(x, y) \in B: x \in\left[1-2^{-k}, 1-2^{-(k+1)}\right)\right\} & \text { if } k \text { is even, } \\
\left\{(x, y) \in B: x \in\left[-1+2^{-(k+1)},-1+2^{-k}\right)\right\} & \text { if } k \text { is odd. }\end{cases}
\end{aligned}
$$

(This partition is closely related to that given in the proof of Theorem 1.)

We define a homeomorphism $\tau \in \mathscr{T}^{2}$ as a product $\tau=\varphi \circ \sigma$ where $\sigma$ is the reflection on the vertical axis, i.e. $\sigma(x, y)=(-x, y)$. The structure of $\varphi$ is more difficult: For all the sets $\sigma\left(S_{k}\right)$, which are of the form $\sigma\left(S_{k}\right)=\left\{(x, y) \in B: x \in\left(a_{k}, b_{k}\right]\right\}$, we consider a covering square $R_{k}=\left[a_{k}-\frac{b_{k}-a_{k}}{3}, b_{k}+\frac{b_{k}-a_{k}}{3}\right] \times\left[-\frac{5\left(b_{k}-a_{k}\right)}{6}, \frac{5\left(b_{k}-a_{k}\right)}{6}\right]$. The closed squares $R_{k}$ are mutually separated. The restriction $\left.\varphi\right|_{R_{k}}$ to $R_{k}$ is a homeomorphism of $R_{k}$ onto itself which leaves the boundary bd $\left(R_{k}\right)$ pointwise fixed and maps $\sigma\left(S_{k}\right)$ onto $T_{k}$. Besides that, we demand the remaining points $(x, y) \notin \bigcup_{k=0}^{\infty} R_{k}$ to be fixed under $\varphi$. It is easily seen that the piecewise definitions of $\varphi$ fit together and form a homeomorphism $\varphi \in \mathscr{T}^{2}$. 


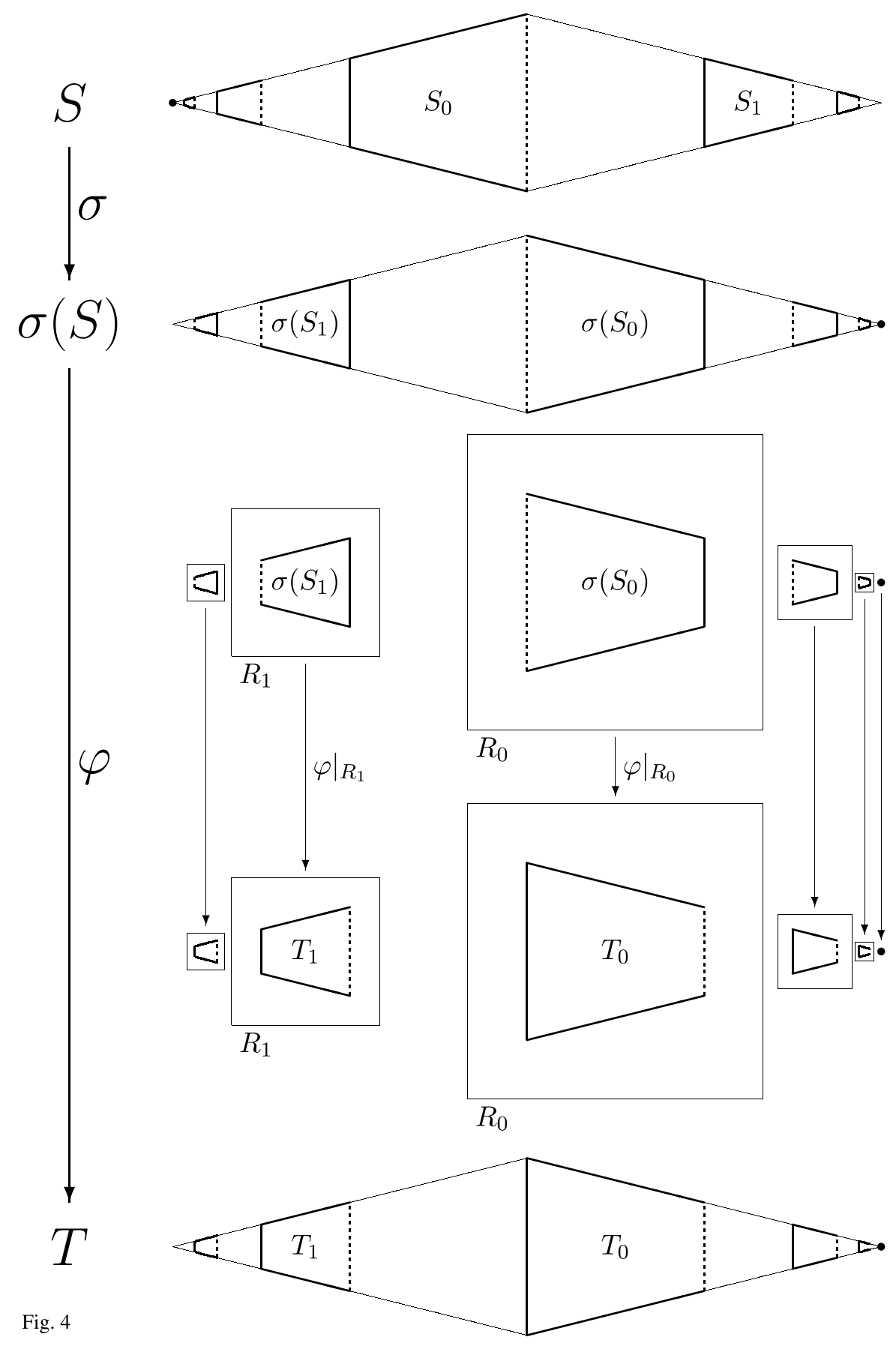


Consequently, we obtain $\tau=\varphi \circ \sigma \in \mathscr{T}^{2}$ such that

$$
\begin{aligned}
\tau(S) & =\varphi \circ \sigma\left(\{(-1,0)\} \cup \bigcup_{k=0}^{\infty} S_{k}\right) \\
& =\varphi(\sigma(\{(-1,0)\})) \cup \bigcup_{k=0}^{\infty} \varphi\left(\sigma\left(S_{k}\right)\right) \\
& =\{(1,0)\} \cup \bigcup_{k=0}^{\infty} T_{k}=T .
\end{aligned}
$$

This completes the verification of Proposition 3.

Proposition 4 Let $d \in\{2,3,4, \ldots\}$ and $n \in\{2,3,4, \ldots\}$ such that a closed ball in $\mathbb{E}^{d-1}$ is $n$-divisible w.r.t. $\mathscr{T}^{d-1}$. Then every closed ball in $\mathbb{E}^{d}$ is $n$-divisible w.r.t. $\mathscr{T}^{d}$.

Proof. According to the assumption there exist a closed ball $B^{d-1} \in \mathbb{E}^{d-1}$, a decomposition $B^{d-1}=S_{1}^{d-1} \cup S_{2}^{d-1} \cup \ldots \cup S_{n}^{d-1}$ of $B^{d-1}$, and mappings $\tau_{i}^{d-1}$ from $\mathscr{T}^{d-1}$ such that $\tau_{i}^{d-1}\left(S_{1}^{d-1}\right)=S_{i}^{d-1}, 1 \leq i \leq n$. Let $B^{d}$ be a closed ball in $\mathbb{E}^{d}$. We assume that $B^{d}=B^{d-1} \times[0,1]$ without loss of generality. Of course, $B^{d}$ admits the decomposition $B^{d}=\left(S_{1}^{d-1} \times[0,1]\right) \cup\left(S_{2}^{d-1} \times[0,1]\right) \cup \ldots \cup\left(S_{n}^{d-1} \times[0,1]\right)$. Moreover, if the identity on $\mathbb{E}^{1}$ is denoted by $\iota$, then the mappings $\tau_{i}^{d}=\tau_{i}^{d-1} \times \iota: \mathbb{E}^{d} \rightarrow \mathbb{E}^{d}, 1 \leq i \leq n$, belong to $\mathscr{T}^{d}$ and fulfil $\tau_{i}^{d}\left(S_{1}^{d-1} \times[0,1]\right)=S_{i}^{d-1} \times[0,1]$. This shows the $n$-divisibility of $B^{d}$ w.r.t. $\mathscr{T}^{d}$.

Propositions 1-4 amount to the following theorem:

Theorem 2 Let $d \in\{1,2,3, \ldots\}, n \in\{2,3,4, \ldots\}$, and let $B$ be a closed ball in $\mathbb{E}^{d}$. Then $B$ is $n$-divisible w.r.t. $\mathscr{T}^{d}$ if and only if $(d, n) \neq(1,2)$.

\section{Partitioning non-closed balls}

Proposition 5 Let $S$ be a subset of $\mathbb{E}^{d}, x_{0} \in \operatorname{bd}(S) \backslash S$ a point such that $S \cup\left\{x_{0}\right\}$ is star-shaped w.r.t. $x_{0}, n \in\{2,3,4, \ldots\}$, and $\delta$ the dilatation with center $x_{0}$ and factor $c>1$. Then there exists a decomposition

$$
S=S_{1} \cup S_{2} \cup \ldots \cup S_{n}
$$

of S such that

$$
S_{i}=\delta^{i-1}\left(S_{1}\right) \quad \text { for } \quad i=1,2, \ldots, n .
$$

Proof. It is sufficient to show the assertion for all intersections

$$
S^{H}=S \cap H
$$

of $S$ with open halflines $H$ starting in $x_{0}$, since $\delta$ maps $H$ onto itself. We assume that $S^{H} \subseteq \mathbb{E}^{1}, x_{0}=0$, and $H=(0, \infty)$ without loss of generality. $S^{H}$ is a bounded or an 
unbounded interval, i.e. $S^{H}=\emptyset, S^{H}=(0, b), S^{H}=(0, b]$, or $S^{H}=(0, \infty)$. If $S^{H}$ is empty then there is nothing to show. Otherwise we consider the decomposition

$$
S^{H}=S_{1}^{H} \cup S_{2}^{H} \cup \ldots \cup S_{n}^{H}
$$

with

$$
S_{i}^{H}= \begin{cases}\bigcup_{k=-\infty}^{-1}\left[b \cdot c^{n k+i-1}, b \cdot c^{n k+i}\right) & \text { if } S^{H}=(0, b), \\ \bigcup_{k=-\infty}^{-1}\left(b \cdot c^{n k+i-1}, b \cdot c^{n k+i}\right] & \text { if } S^{H}=(0, b], \\ \bigcup_{k=-\infty}^{\infty}\left(c^{n k+i-1}, c^{n k+i}\right] & \text { if } S^{H}=(0, \infty) .\end{cases}
$$

A simple calculation shows that

$$
S_{i}^{H}=c^{i-1} \cdot S_{1}^{H}=\delta^{i-1}\left(S_{1}^{H}\right)
$$

for $i=1,2, \ldots, n$ and completes the proof.

Of course, for every strictly convex set $S \subseteq \mathbb{E}^{d}$, which is not closed, there exists a point $x_{0} \in \operatorname{bd}(S) \backslash S$ as assumed in Proposition 5. Hence we obtain:

Corollary Every non-closed strictly convex set in $\mathbb{E}^{d}$ is n-divisible w.r.t. the group ged of all similarities of $\mathbb{E}^{d}$ for $n=2,3,4, \ldots$.

In the context of the present paper we formulate the following more special implication concerning the divisibility of non-closed balls w.r.t. $\mathscr{T}^{d}$ :

Theorem 3 Let $d \in\{1,2,3, \ldots\}, n \in\{2,3,4, \ldots\}$, and let $B$ be a non-closed ball in $\mathbb{E}^{d}$. Then $B$ is n-divisible w.r.t. $\mathscr{T}^{d}$.

Let us close this paper with a remark on the structure of the decompositions of closed or open balls in $\mathbb{E}^{1}$ considered in the proofs of Proposition 2 and Proposition 5. The balls are partitioned into subsets mutually congruent via $\mathscr{T}^{1}$, any of the subsets consisting of countably many connected components. The partitions given in this paper are optimal in the following sense:

Proposition 6 Let $B=S_{1} \cup S_{2} \cup \ldots \cup S_{n}$ be a decomposition of a closed or open ball $B \in \mathbb{E}^{1}$ into $n \geq 2$ subsets which are pairwise congruent via $\mathscr{T}^{1}$. Then all the sets $S_{i}$ consist of infinitely many connected components.

Proof. We assume the contrary, i.e. the boundaries $\operatorname{bd}\left(S_{i}\right)$ of the subsets $S_{i}$ w.r.t. the topology of $\mathbb{E}^{1}$ are finite. Then the interiors of the sets $S_{i}$ consist of $k$ open intervals, where $k \geq 1$ does not depend on $i$ according to the congruence of the sets $S_{i}$ via $\mathscr{T}^{1}$. Hence the ball $B$ is decomposed into $n k$ open intervals - the components of $\operatorname{int}\left(S_{i}\right)$, $1 \leq i \leq n,-$ and those points of the boundaries of the sets $S_{i}$ which belong to $B$. The $n k$ intervals are separated by $n k-1$ inner points of $B$. Consequently,

$$
\operatorname{card}\left(\bigcup_{i=1}^{n}\left(\operatorname{bd}\left(S_{i}\right) \cap B\right)\right)= \begin{cases}n k+1 & \text { if } B \text { is a closed interval, } \\ n k-1 & \text { if } B \text { is an open interval. }\end{cases}
$$


On the other hand we have

$$
\bigcup_{i=1}^{n}\left(\operatorname{bd}\left(S_{i}\right) \cap B\right)=\bigcup_{i=1}^{n}\left(\operatorname{bd}\left(S_{i}\right) \cap S_{i}\right)
$$

where the right union is disjoint. All the sets $\operatorname{bd}\left(S_{i}\right) \cap S_{i}, 1 \leq i \leq n$, are of the same cardinality $l$, since the sets $S_{i}$ are congruent via $\mathscr{T}^{1}$. Thus we obtain

$$
\operatorname{card}\left(\bigcup_{i=1}^{n}\left(\operatorname{bd}\left(S_{i}\right) \cap B\right)\right)=n l
$$

This contradiction to formula (3) proves Proposition 6.

The author thanks E. Hertel for confronting him with the problem and for encouraging him to write this paper.

\section{References}

[Ede1] M. Edelstein: Isometric decompositions, J. London Math. Soc. 37 (1988), 158-163

[Ede2] M. Edelstein: On the indecomposability of compact convex sets, Proc. Amer. Math. Soc. 115 (1992), 737-739

[E/J/T] M. Edelstein, K. Johnson, A. C. Thompson: On the isometric dissection problem for convex sets, Studia Sci. Math. Hungar. 27 (1992), 273-277

[He] E. Hertel: Disjunkte Pflasterungen konvexer Körper, Studia Sci. Math. Hungar. 21 (1986), 379-386

[Ri] C. Richter: On decompositions of compact convex sets, Geom. Dedicata 71 (1998), 1-4

[Wae] B. L. van der Waerden: Aufgabe 51, Elem. Math. 4 (1949), 18

[Wa1] S. Wagon: Partitioning intervals, spheres and balls into congruent pieces, Canad. Math. Bull. 26 (3) (1983), 337-340

[Wa2] S. Wagon: The Banach-Tarski paradox, Cambridge Univ. Press, 1986

Christian Richter

Friedrich-Schiller-Universität

Ernst-Abbe-Platz 1-4

D-07743 Jena

Germany 